\title{
LA-UR-21-22922
}

Approved for public release; distribution is unlimited.

Title: $\quad$ Experiments at TA-55 have used optical pyrometry to measure temperatures of shocked plutonium on the $40 \mathrm{~mm}$ gun

Author(s): $\quad$ Jensen, Brian J.

Dattelbaum, Dana Mcgraw

Intended for: marketing, program management updates

Issued: $\quad 2021-03-25$ 
Disclaimer:

Los Alamos National Laboratory, an affirmative action/equal opportunity employer, is operated by Triad National Security, LLC for the National Nuclear Security Administration of U.S. Department of Energy under contract 89233218CNA000001. By approving this article, the publisher recognizes that the U.S. Government retains nonexclusive, royalty-free license to publish or reproduce the published form of this contribution, or to allow others to do so, for U.S. Government purposes. Los Alamos National Laboratory requests that the publisher identify this article as work performed under the auspices of the U.S. Department of Energy. Los Alamos National Laboratory strongly supports academic freedom and a researcher's right to publish; as an institution, however, the Laboratory does not endorse the viewpoint of a publication or guarantee its technical correctness. 


\section{Experiments at TA-55 have used optical pyrometry to measure temperatures of shocked plutonium on the $40 \mathrm{~mm}$ gun}

Researchers at LANL, along with collaborators from MSTS, have worked for more than a decade to develop and implement an optical pyrometry method to measure the temperature of metals shocked to the high pressures and temperatures. Because traditional shock wave measurements only provide information on the mechanical state of the material, temperature measurements are required to validate and improve equation-of-state (EOS) models for materials. The conditions sampled are complex; commenserate with those found in planetary impacts, and accressed by conventional and nuclear weapons. Optical pyrometry is currently the best available method for inferring the temperature of shocked metals, by careful measurement of the calibrated radiance emitted by a surface at finite temperature. These measurements are difficult often complicated by the short time scales associated with shock wave experiments $(<1 \mu \mathrm{s})$ coupled with the many sources of non-thermal light (impact flash, fracture light, etc.) that pollutes the radiant light measured from the sample.

\section{First successful temperature measurement at TA-55}

Over 2 years, the TA-55 pyrometry team executed a plan of work to qualify the Compact-6 pyrometer, complete prove-out experiments at LANL's Dynamic Equation-ofState (DEOS) facility on other metals, and implement the system within TA-55. In July of 2020 , the Compact- 6 was installed in the control room, followed by the first dynamic radiance measurement on plutonium in November 2020. This work represents a multi-year effort to meet an FY21 Level 2 milestone for the Dynamic Materials Properties Program, and supports a collaborative tri-lab temperature measurement effort. The ability to routinely measure temperature in shock metals represents a significant step forward in efforts validate multi-phase equations of state, and to modernize the LANL's TA-55 40mm gun - a signature national facility for plutonium studies. The data obtained will have a significant impact on our predictive capabilities for simulating weapon physics performance.
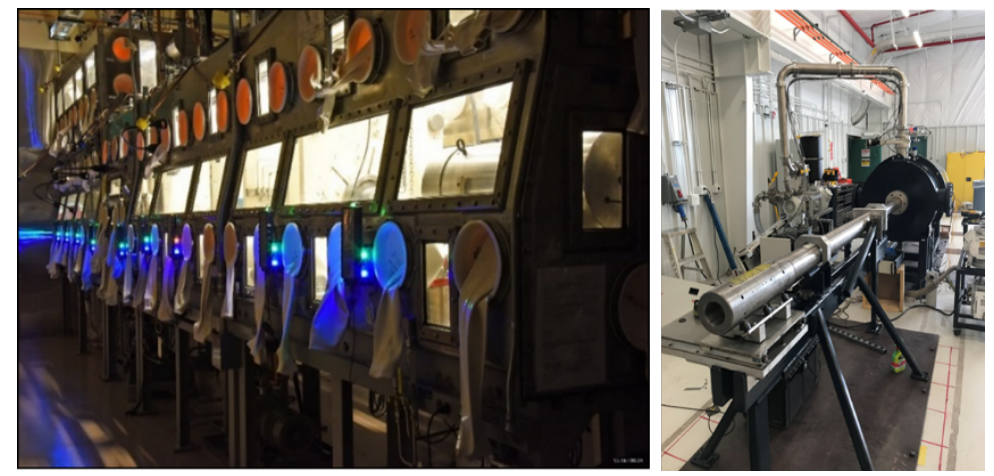

Figure 1. Photo of the TA-55 $40 \mathrm{~mm}$ gun and glove box (Left) and the $40 \mathrm{~mm}$ powder gun at DEOS (right). The DEOS $40 \mathrm{~mm}$ gun is the test bed for developing new experiments and diagnostics prior to being conducted on the TA-55 40mm gun.
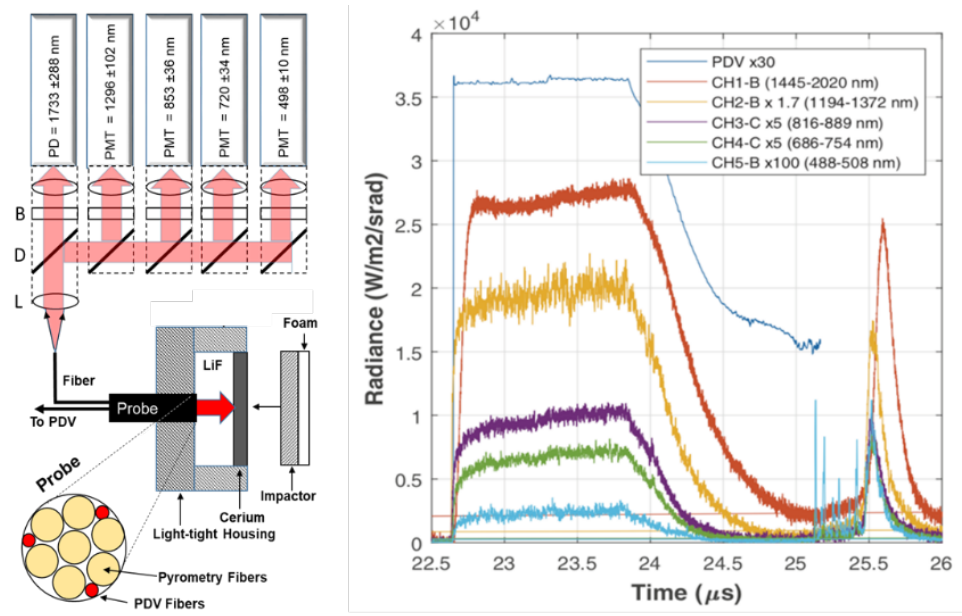

Figure 2. Example optical pyrometry experiment. (Left) Experiment configuration showing an impactor impacting a Ce sample. A multi-fiber pyrometry and velocimetry probe couples light to a multi-channel pyrometer to measure the radiant light from the Ce-LiF interface. (Right) Published radiance data and velocimetry shown on the right for shocked cerium. Phys Rev. B. 102, 214105 (2020)

Contact:

Dynamic Materials

Properties Program

Dana Dattelbaum

danadat@lanl.gov

(505) 500-2070
Brian Jensen

Principal Investigator

bjjensen@lanl.gov

(505) 500-2802 